\title{
A Device Monitoring System Based on ARM Upper Computer and WIFI Transmission
}

\author{
Mingbo Zhao, Song Yan, Qingrui Miao \\ Department of Computer Science, Shandong University of Technology, Zibo, China \\ Email: zhaomingbosdut@qq.com
}

How to cite this paper: Zhao, M.B., Yan, S. and Miao, Q.R. (2019) A Device Monitoring System Based on ARM Upper Computer and WIFI Transmission. Journal of Computer and Communications, 7, 72-82. https://doi.org/10.4236/jcc.2019.79006

Received: August 26, 2019

Accepted: September 21, 2019

Published: September 24, 2019

Copyright $\odot 2019$ by author(s) and Scientific Research Publishing Inc. This work is licensed under the Creative Commons Attribution International License (CC BY 4.0).

http://creativecommons.org/licenses/by/4.0/

\section{(c) (i) Open Access}

\begin{abstract}
With the widespread application of WIFI networks and embedded system technology, the device monitoring system based on Embedded System and wireless network came into being. In this paper, we introduce a device monitoring system based on ARM upper computer and WIFI transmission, and we tested this system on workshop equipment. The hardware adopts ARM Cortex-A8 processor architecture of TI company as the main control chip, using IAC-335X-Kit development board for system design, external USB camera module and WIFI wireless module for video capture and data transmission. The software is based on embedded Linux as the platform. The system will collect production data accurately and objectively, and the statistical analysis. At the same time, the system uses QT to develop the upper computer software GUI interface. Compared with the traditional system based on the wired network, our design is more convenient and flexible, which reduces the implementation restriction and maintenance cost of traditional network cabling.
\end{abstract}

\section{Keywords}

ARM Upper Computer, WIFI Transmission, Embedded System, QT

\section{Introduction}

With the development of wireless network technology, the transmission of network information becomes more secure and convenient. WIFI has become the mainstream standard of wireless network access because of its wide coverage, high reliability, fast speed, no wiring, health and safety. And with the increasing demand for applications based on WIFI technology, more and more electronic products will provide WIFI wireless access function, which will have a very far-reaching impact on the application of wireless WIFI technology [1] [2]. 
Based on the above understanding of WIFI transmission technology, Our main contribution is that the system is based on the maturity of wireless network technology and the universal application of various sensors to reduce the implementation limitation of traditional wired network communication, and the system is applied to verify in the new environment of workshop, in order to increase the hands of enterprises or workshop managers on production. Control ability improves production efficiency and enterprise profit.

The main technologies involved are video acquisition and transmission and WIFI wireless communication. Video capture device uses common USB interface cameras to connect directly to the ARM server. Video frame acquisition is realized through the interface provided by V4L2 driven by Linux kernel, and real-time display is achieved after data format conversion [3] [4]. At the same time, FFMPEG open source library is used to compress video, generate common video formats, facilitate viewing and play them on various devices. With regard to WIFI wireless communication, the wireless network card with USB interface is inserted into the ARM development board in hardware. WIFI network architecture is a centralized network, that is, through the fixed infrastructure AP as the central station, all client devices and ARM server communicate through AP [5]. The ARM server provides the modification operation of parameters and sends it to the designated client (device), at the same time through WIFI to simulate the control of the device [6].

The rest of this paper is organized as follows: in Section 2, we describe our overview of system design. Section 3 presents the system software design in detail, which includes the function design of wireless communication module, the design of database structure and function and the software interface design of upper computer. Section 4 shows the implementation details of the system. In Section 5, we discuss the system operation and testing. In Section 6, we review related work and the conclusion is drawn in Section 7.

\section{Overview of System Design}

The design structure of the system is shown in Figure 1. The system takes WIFI wireless transmission technology as the core and equipment data parameters as the basis to realize the monitoring and control of workshop equipment. The design focuses on the development of the upper computer software on the ARM server side, as well as the wireless transmission and processing of data. The acquisition of equipment parameter data is simulated by software, that is, through the PC-side software simulation equipment, connected to the ARM server through WIFI, and sent the processed random number simulation equipment parameters regularly. The ARM server displays and saves the received devices to the database in real time after data processing, and provides queries for summary information.

In hardware, the ARM server connects video acquisition device and wireless network card device through USB interface, realizes video acquisition and wireless communication based on WIFI. The WIFI network adopts central network 
architecture and uses wireless AP as access point to realize wireless communication between equipment and server.

\section{System Software Design}

Wireless communication adopts client-server communication mode based on $\mathrm{C} / \mathrm{S}$ architecture. Devices as client clients are simulated by PC software, and ARM servers are servers. TCP protocol is used to realize network communication [7]. When the program starts running, the server opens the listening port and runs separately in threads, waiting for the connection of the client device.

As shown in Figure 2, Wireless Communication Flow Chart is described. The following is an explanation of the flow chart.

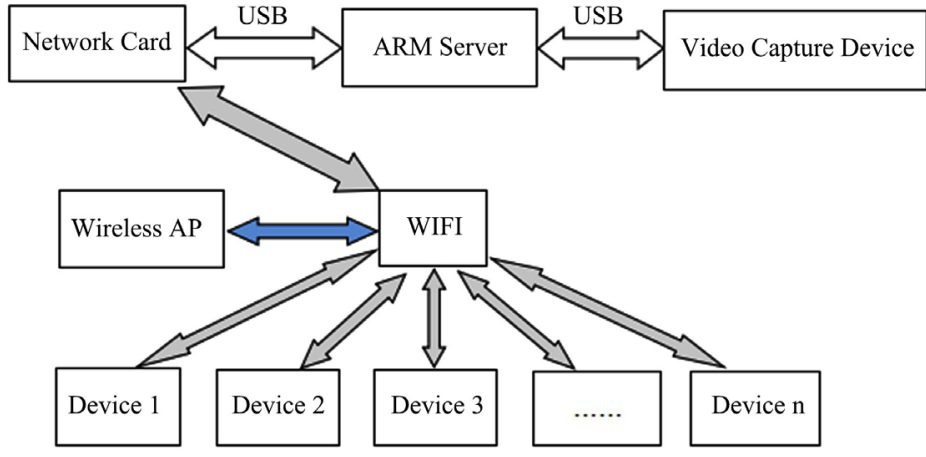

Figure 1. System design block diagram.

Client (device)

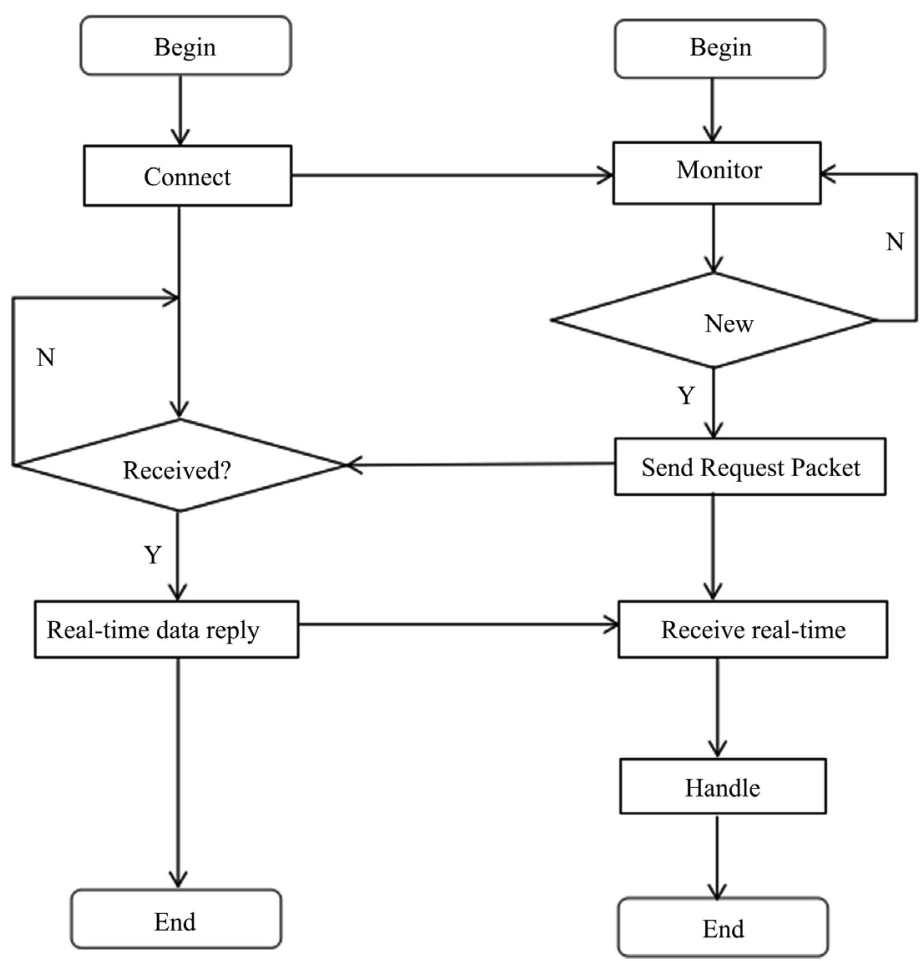

Figure 2. Wireless communication flow chart. 
The PC-side software simulates the client device and initiates the connection to the ARM server. When the server receives the connection request from the client, it will save the client in the list and send the command of requesting data to the client in the list regularly. Request commands are encapsulated and packaged by HDLC protocol customized by the company. After the client receives the request data from the server, the original request command is decomposed according to HDLC protocol, and its checkout and control commands are judged. If correct, the client replies real-time data to the server. Otherwise, it will continue to wait for the request of the server.

The real-time data returned by the client is also encapsulated by HDLC protocol. The server decomposes and judges the received real-time data. When the transmission is correct, the real-time data is converted into intuitive data to display and save. In addition, the real-time data sent by each client includes not only the device parameters, but also the corresponding IP address of the device and the corresponding time of the data, so as to realize the synchronous monitoring and control of multiple devices by the server.

Next, we introduce our database structure and function design, which mainly includes database logical design and database physical design. As an open source embedded database product, SQLite has the characteristics of low system overhead and high retrieval efficiency. At the same time, it is a zero-configuration database, without installation and management. A complete SQLite database is stored on a single disk file, self-sufficient, without any external dependencies. In addition, the development of the QT framework used to support SQLite is better, so the final database selected SQLite. Figure 3 and Figure 4 are based on the Entity-Relation model (ER model) of the database. Two entity models are designed, namely, workshop equipment information entity and real-time equipment information entity. According to the logical design of the above database, the entity relationship is transformed into data table, which can be divided into two tables to complete the physical design of the database. Table 1 and Table 2 correspond to Figure 3 and Figure 4 respectively. Through the above structure design of the database, it can provide good background data support for the system. The workshop equipment information table provides the addition, deletion and modification of basic equipment information, while the equipment real-time information table provides the storage and display of real-time production data, and its statistical analysis, in order to improve the efficiency of enterprise production management.

As a cross-platform C++ GUI library, Qt is developed by Troll Tech, Norway. With its object-oriented, easy to expand, component programming and cross-platform features, Qt is widely used in GUI interface development of embedded systems. Excellent cross-platform characteristics, programs written using Qt class can be compiled by different compilers to generate programs running on different platforms, which greatly reduce the difficulty of cross-platform development. Therefore, this design uses the embedded version of $\mathrm{Qt}$, that is, the 


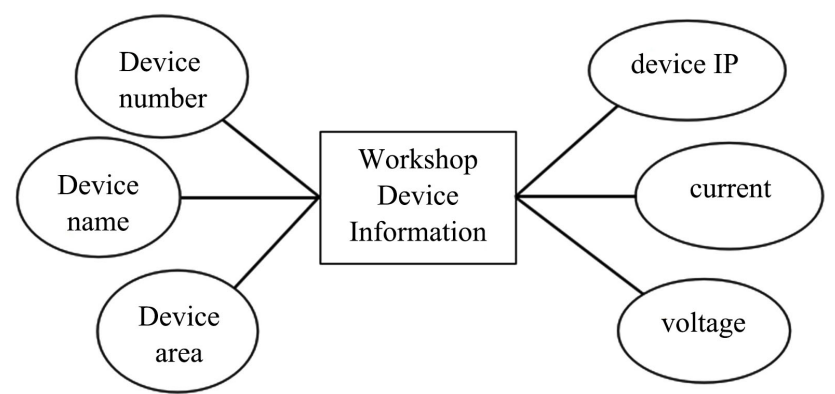

Figure 3. E-R Diagram of workshop device information entity.

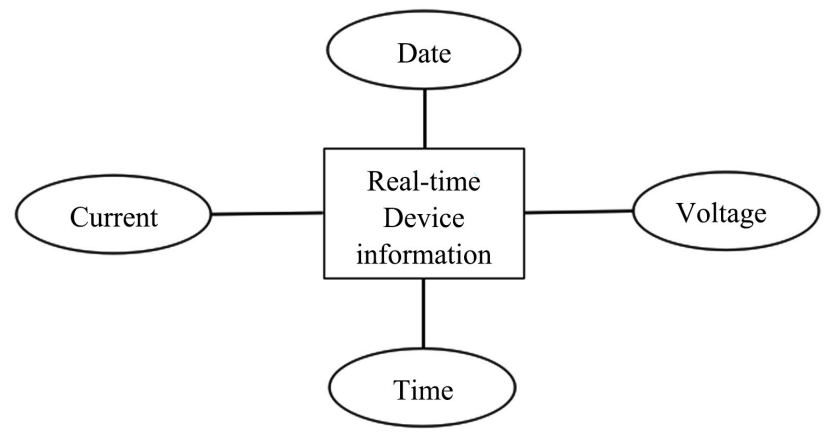

Figure 4. E-R Diagram of device real-time information entity.

Table 1. Workshop equipment information table.

\begin{tabular}{|c|c|c|c|c|c|c|}
\hline Primary & Field name & Data type & Length & Accuracy & Unique & Noempty \\
\hline & No & Varchar & & & Yes & Yes \\
\hline & Area & Varchar & & & & \\
\hline & Name & Varchar & & & & \\
\hline & IpAddr & Varchar & & & Yes & \\
\hline & PresetA & Int & & & & \\
\hline & PresetV & Float & & & & \\
\hline
\end{tabular}

Table 2. Equipment real-time information table.

\begin{tabular}{|c|c|c|c|c|c|c|}
\hline Primary & Field name & Data type & Length & Accuracy & Unique & Noempty \\
\hline & Edate & Date & & & & \\
\hline & Etime & Time & & & & \\
\hline & Evoltage & Float & & & & \\
\hline & Ecurrent & Int & & & & \\
\hline
\end{tabular}

Qt/Embedded development framework based on Frame buffer to develop the upper computer software interface [8] [9].

As shown in Figure 5, the upper computer software interface is divided into four major modules, workshop general situation, information display, informa- 
tion summary and network settings. Through these four modules, the whole system monitoring and control flow from network to data acquisition, control command sending, display and statistical analysis is realized. Combined with the component programming characteristics of QT, the main interface uses the component of $\mathrm{Q}$ Tab Widget to realize the switch of four modules. According to the object-oriented characteristics of $\mathrm{C}++$, the four modules inherit from $\mathrm{Q}$ Widget as an independent interface class, encapsulate the sub-components needed by the module, and complete different information display.

\section{Implementation}

The following content is to set up four modules of the whole interface through tabWidget. Among them, the four module interfaces inherit from QWidget as a separate interface class. All of them are used to encapsulate the sub-components needed by the module and complete different information display.

TabWidget->setFocusPolicy(Qt::NoFocus);//The aim is not to set the focus and avoid dotted boxes

TabWidget->setTabPosition(QTabWidget::South);//Settings are placed below the interface

TabWidget->setFont(QFont("msyh",16));

PlantGlobal Widget=new PlantGlobal Widget(this);

TabWidget->addTab(plant global widget,tr("workshop status");//The following four sentences describe the four modules

InfoShowWidget=new InfoShowWidget(this);

TabWidget->addTab(infoShowWidget,tr("Information Display");

InfoCollectWidget=new InfoCollectWidget(this);

TabWidget->addTab(infoCollectWidget,tr("Information Summary");

Networking Widget=new Networking Widget(this);

TabWidget->addTab(networking widget,tr("network settings");//Initialization server sends instruction packages of request data to connected clients at regular intervals. The instruction data is the same

Void initRequestData();

//Data is processed by HDLC protocol in the company during network transmission

QByteArray data to HDLC(QByteArray data);

Void HDLCToData(QByteArray\&enciphered Data);

QStringList convertReceive Message(QByteArray\&array);

//The goal is to get the QTcpSocket connected to the server, that is, the socket created by the client

Void getClientSocket();

Void receiveMessage();//The server receives messages from the client

Void sendMessage();//The server sends a message requesting data

Void sendMessage Data(QStringList);//The server replies to data

Void disconnectSlot();//Signal Processing for Disconnecting Client 


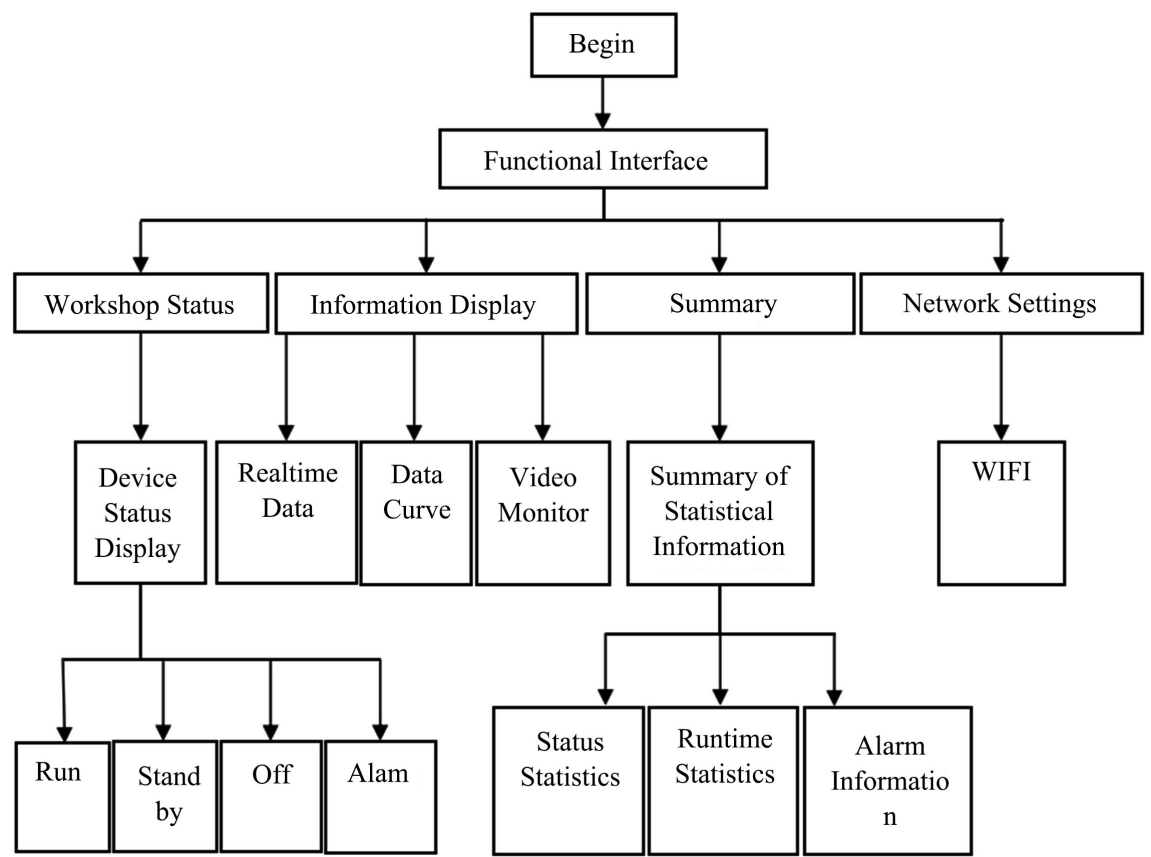

Figure 5. Software interface structural diagram.

\section{System Operation and Testing}

We run experiments with the following configurations:

1) Hardware:

Windows PC, Hangzhou Qiyang IAC-335X-Kit ARM development/evaluation board, QY-AT070TN83 LCD touch screen, USBHub hub, USB interface video acquisition module, RT3070 USB wireless network card.

2) Software and development environment:

Qt for Windows development tools, Ubuntu 14.04, Keil for arm, Sqlite 3 database, Qt 4.8.2 for ARM embedded development environment, tftpad 32 burner.

The physical connection of our hardware is shown in Figure 6. This design uses the IAC-335X-Kit development/evaluation board provided by Hangzhou Qiyang Company, which is showed in Figure 7. The IAC-335X-MB base plate, which is the standard of floor expansion, adopts high-precision four-layer board technology, has the best electrical and anti-interference capability, and fully expands the various interface resources supported by AM335X [10] [11]. And our Specific test contents and results of workshop general situation interface, specific test contents and results of information display interface, specific test contents and results of information summary interface correspond to Tables 3-5 below respectively.

\section{Related Work}

Nowadays, WIFI transmission has different applications in various fields. Guo et al. thought that wireless transmissions are a potentially powerful and widely available source of transmissions for passive radar detection, and this work shows 
that this technique has considerable promise for a low cost and widely deployable detection and tracking system [12]. Xue et al. presented a new ARM + GPRS structure which can apply in the orchard soil information collection and transmission. It is a design of data acquisition and wireless transmission control system based on ARM9, and it has been proved by experiments that the system has features of small size, high performance, real-time and low-cost [13]. For enterprises, timely and accurate acquisition of workshop production equipment data and production environment is an important aspect for enterprises to make full use of information resources, improve management level and move towards information technology. Therefore, with the widespread application of all kinds of sensors, it has become a trend to monitor and control workshop equipment through WIFI technology [14].

Table 3. Workplace general situation interface function test table.

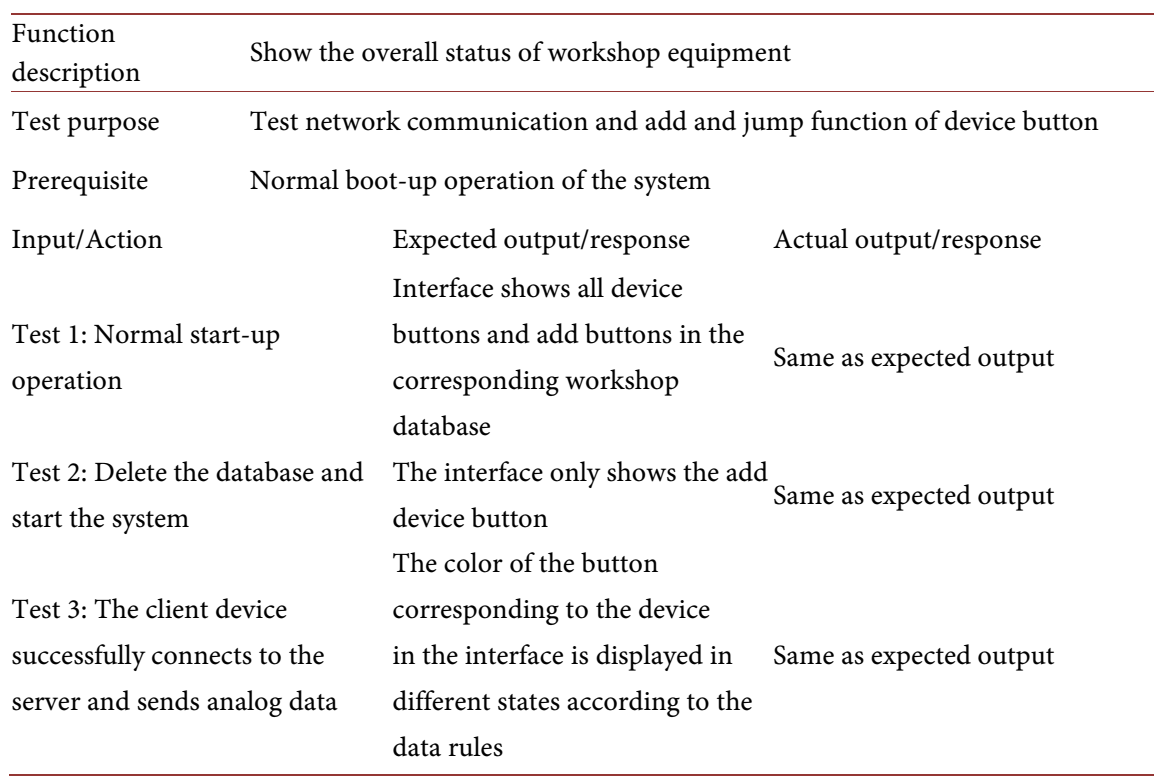

Table 4. Information display interface function test table.

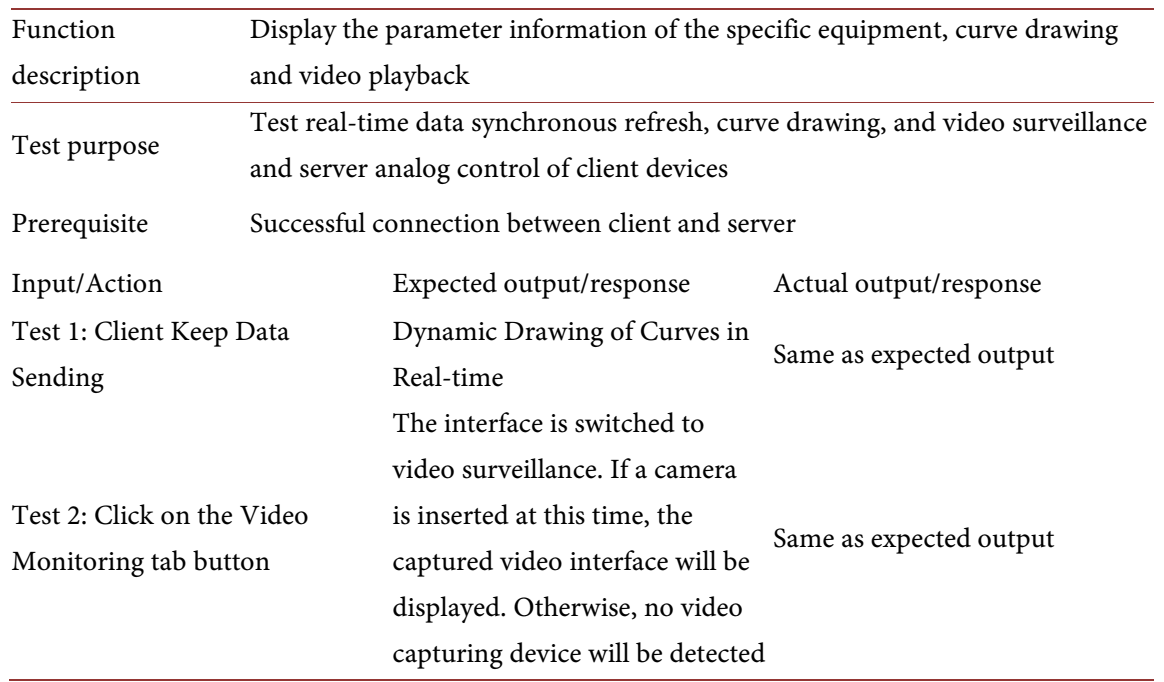


Table 5. Information summary interface function test table.

\begin{tabular}{|c|c|c|c|}
\hline $\begin{array}{l}\text { Function } \\
\text { description }\end{array}$ & \multicolumn{3}{|c|}{$\begin{array}{l}\text { Statistical inquiry of workshop equipment status, running time and alarm } \\
\text { information }\end{array}$} \\
\hline Test purpose & \multicolumn{3}{|c|}{$\begin{array}{l}\text { Real-time Synchronization of Test Equipment Status and Writing and Query } \\
\text { of Database }\end{array}$} \\
\hline Prerequisite & \multicolumn{3}{|c|}{ Normal operation of program } \\
\hline Input/Action & & Expected output/response & Actual output/response \\
\hline \multicolumn{2}{|c|}{$\begin{array}{l}\text { Test 1: Change the state of a } \\
\text { device }\end{array}$} & $\begin{array}{l}\text { Change of the corresponding } \\
\text { state in the statistical region of } \\
\text { equipment status }\end{array}$ & Same as expected output \\
\hline \multicolumn{2}{|c|}{$\begin{array}{l}\text { Test 2: In the alarm information } \\
\text { statistics interface, select the } \\
\text { specified date and equipment } \\
\text { number, and click on the query }\end{array}$} & $\begin{array}{l}\text { List the corresponding alarm } \\
\text { records of the equipment below, } \\
\text { and give prompts if there is no } \\
\text { data record }\end{array}$ & Same as expected output \\
\hline
\end{tabular}

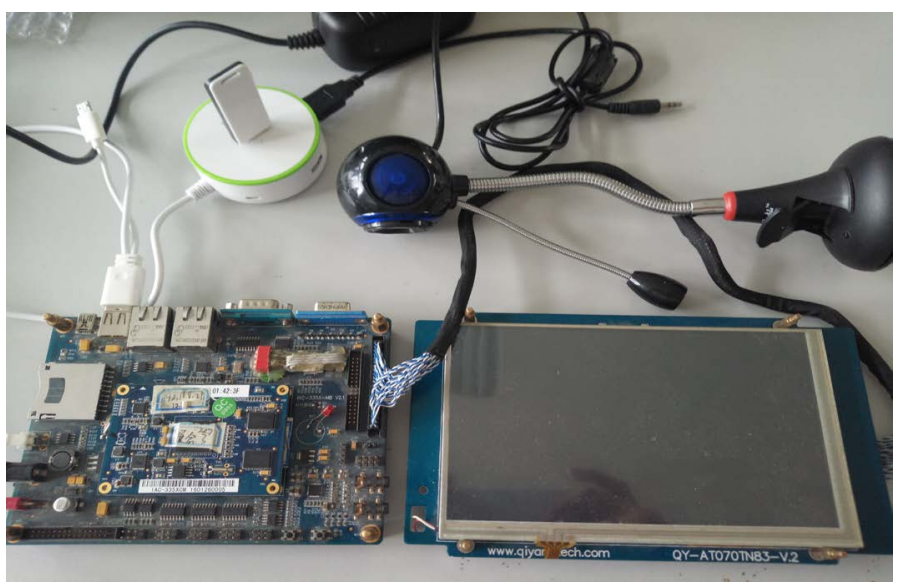

Figure 6. Hardware physical connection diagram.

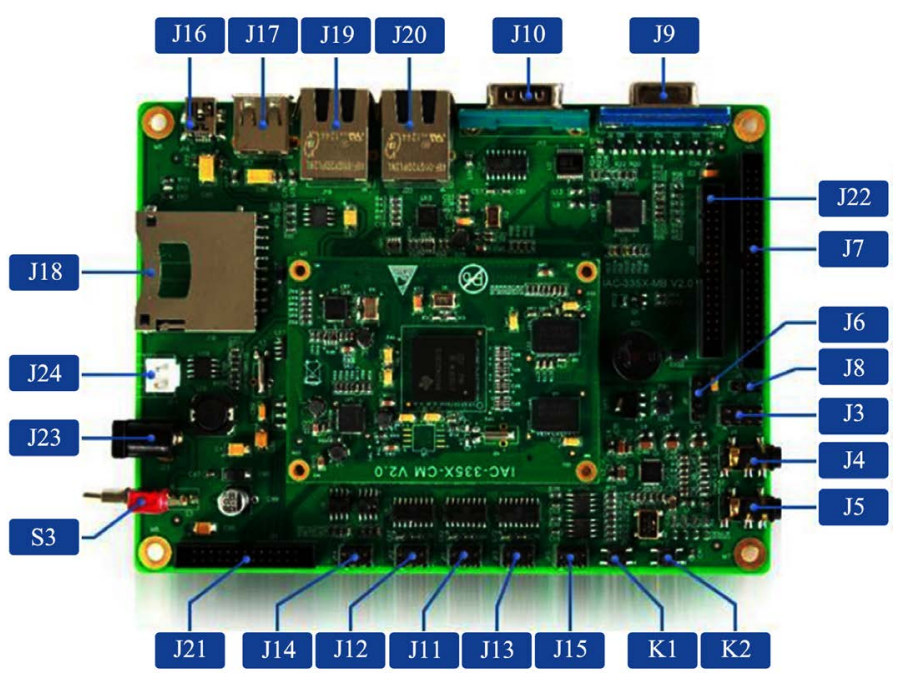

Figure 7. Development board baseboard resource interface diagram.

\section{Conclusion}

In this paper, the system uses ARM processor as the main control chip, embed- 
ded Linux system as the software platform, and uses QT to develop the GUI interface of host computer software. Compared with similar products, this design has the advantages of using embedded system as a server, low power consumption, small size, high integration, low cost, customizable, tailorable and a series of other advantages make it more suitable for factory workshop production environment. In addition, WIFI wireless communication is more convenient and flexible for data transmission. Compared with the monitoring and control system based on wired network, it reduces the implementation limitation and maintenance cost of traditional network wiring and reduces the cost investment of enterprises.

\section{Conflicts of Interest}

The authors declare no conflicts of interest regarding the publication of this paper.

\section{References}

[1] Li, H., Gao, Z.H., Gao, F. and Zhao, R.H. (2009) Research on IEEE 802.11 Wireless LAN Standard. Computer Application Research, 26, 1616-1620.

[2] Zhang, Y., Liu, Y.P. and Qi, G.F. (2010) Brief Discussion on WIFI Wireless Coverage Technology and Its Realization. Information System Engineering, 6, 60-61.

[3] Ding, G.H. and Hu, R.Q. (2004) Socket Programming of Linux and Its Application in Embedded Gateway. Application of Electronic Components, 10, 21-24.

[4] Xu, Q.Q. (2009) Exploration and Programming of USB Camera Based on Linux. Science and Technology Innovation Report, 36, 13-14.

[5] Blabchette, J. and Summerfield, M. (2008) C + + GUI Qt4 Programming. Electronic Industry Press, Beijing.

[6] Zhang, X.L. and Cui, Y.W. (2006) Embedded System Design and Practice. University of Aeronautics and Astronautics Press, Beijing.

[7] Ling, K. and Leng, J.W. (2016) Design of Embedded Video Surveillance System Based on WiFi. Electronic Engineering Design, 24, 177-179.

[8] Huo, Y.F. (2014) Quick Start of Qt Creator. Beijing University of Aeronautics and Astronautics Press, Beijing.

[9] Yu, B.W. and Deng, H.Q. (2011) Implementation of Control Interface of Embedded Digital Monitoring System Based on Qt/Embedded. Television Technology, 35, 25-28.

[10] Xue, Y. and Liu, W. (2011) Design and Implementation of a Multi-Channel Real-Time Data Acquisition and Monitoring System. Computer Measurement and Control, 19, 863-865.

[11] Xu, L. (2012) Research and Implementation of Embedded Wireless Video Monitoring System Based on ARM11. PhD Thesis, Wuhan University of Science and Technology, Wuhan.

[12] Guo, H., Woodbridge, K. and Baker, C.J. (2008) Evaluation of WiFi Beacon Transmissions for Wireless Based Passive Radar. IEEE Radar Conference, Rome, 26-30 May 2008, 1-6. https://doi.org/10.1109/RADAR.2008.4720810

[13] Xue, H., Gao, L., Li, W., Liu, J., Zhang, J. and Yang, L. (2010) ARM9-Based 
Real-Time Acquisition of Orchard Soil Information and GPRS Wireless Transmission Control System. 2nd International Conference on Future Computer and Communication, Wuha, 21-24 May 2010, 594-598.

https://doi.org/10.1109/ICFCC.2010.5497540

[14] Zhang, Q.Z. and Shen, J.H. (2006) Research and Design of ARM \& Linux Embedded System Boot Loader. Computer Applications and Software. 